\title{
Opioid surveillance: monitoring and responding to the evolving crisis
}

\author{
Kahina Abdesselam, MSc; Matthew James Dann, MSc; Ramona Alwis, MSc; Julie Laroche, PhD; \\ Samuel Ileka-Priouzeau, MSc
}

Tweet this article

\begin{abstract}
The incidence of opioid-related overdoses is increasing at an alarming pace, largely driven by the increased use of fentanyl and its analogues. The need for sound and reliable sources of data on opioid use is crucial in order to make decisions on implementing efficient interventions, and develop appropriate policies and guidelines to mitigate the burden of opioid use.

This article highlights initiatives undertaken by federal partners to address the opioid crisis in Canada. The need for novel surveillance approaches that improve the collection and harmonization of drug-related data is also discussed.
\end{abstract}

Keywords: opioid, fentanyl, surveillance, national trends, drug misuse

\section{Background}

The incidence of opioid-related overdoses continues to increase at an alarming pace, largely driven by the increased use of fentanyl and its analogues. There were 2800 opioid-related deaths in Canada in 2016, and preliminary data suggest that the number of deaths surpassed 4000 in 2017. ${ }^{1}$ From a public health standpoint, this is a major crisis, as the number of deaths in 2016 exceeded the number at the height of the HIV epidemic in Canada in $1995 .^{2}$ The sheer number of overdoses related to fentanyl and its analogues suggests that opioid misuse affects all Canadians, not only people living on the fringes of society. ${ }^{3}$

Per capita, Canada is the second largest consumer of opioids. ${ }^{4}$ A recent opinion poll from the Angus Reid Institute revealed that 1 in 5 adult Canadians have been prescribed opioids. ${ }^{5}$ In addition, 1 in 8 adult Canadians say they have close friends or family members who have become dependent on opioids in the last 5 years. ${ }^{5}$ Although the number of opioid prescriptions dispensed across the provinces increased slightly $(2.7 \%$, populationadjusted) from 2012 to 2016, there was a sharp reduction (34.2\%, populationadjusted) from 2016 to 2017. Similarly, the total morphine milligram equivalents (MME) dispensed between 2012 and 2016 declined slightly. The sharp reduction between 2016 and 2017 (15.9\%) could indicate a change in opioid-prescription practices by physicians. ${ }^{6}$

Prescription trends across Canada paint a heterogeneous picture of the type, quantity and strength of opioids dispensed across the country (Figure 1). Moreover, an upward trend in emergency department visits and hospitalizations due to opioid overdoses (30\% increase between 2007/2008 and $2014 / 2015^{7}$ ) suggests that opioid misuse is an issue of growing concern and that people who use these substances may be increasingly turning to the illicit market.

While national-level surveys of the general population are usually well-developed, data on subpopulations susceptible to substance misuse are lacking. This hampers the ability to develop targeted, timely and
Highlights

- The number of opioid prescriptions and morphine milligram equivalents (MMEs) prescribed sharply declined in all provinces in 2016 and 2017.

- Federal partners undertook several initiatives to better understand Canada's opioid crisis.

- Current surveys of the general population should be complemented by newer innovative surveillance approaches that are better able to target people who use drugs.

appropriate evidence-based drug policies and interventions aimed at tackling the current crisis. For example, the Canadian Tobacco, Alcohol and Drugs Survey (CTADS) and similar surveys (see Table 1) offer some insight on the opioid burden at a national level. However, general population surveys provide limited information on key indicators, such as the reasons for fluctuations in prevalence of opioid use; the types of opioids being used; where opioids are being used; and many sociodemographic characteristics, such as socioeconomic status, age, sex, ethnicity, housing, etc. Thus, the behavioural and risk patterns of individuals susceptible to opioid misuse may be significantly underrepresented due to the sampling methodology used in these population surveys. ${ }^{8}$

Enhanced surveillance systems and studies specifically tailored to gather information on hard-to-reach populations are required to better understand the underlying mechanisms associated with opioid misuse. These can also help highlight 


\section{FIGURE 1}

Overall opioids ${ }^{\mathrm{a}}$ dispensed by pharmacies (per 10000 population), along with their morphine milligram equivalents (MME) per person per day, provinces, ${ }^{b}$ Canada, $2017^{c 7}$

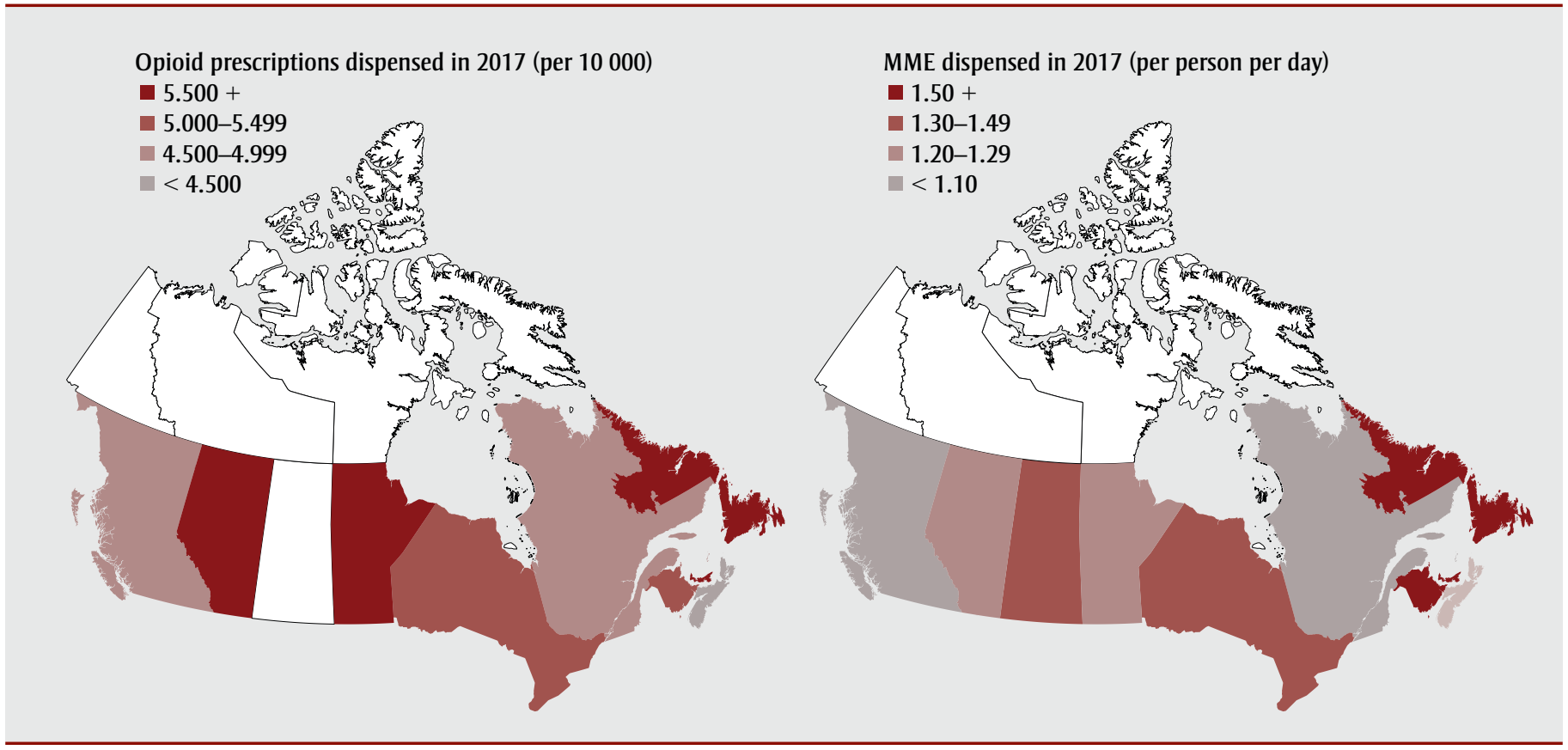

Source: IQVIA. Compuscript. 2017.

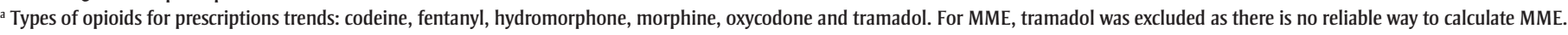

${ }^{\mathrm{b}}$ Rates for Prince Edward Island and Newfoundland and Labrador are combined.

' January to November 2017 only, as December 2017 data had not been added to the database at the time of extraction.

TABLE 1

Overview of the existing and new federal surveillance initiatives that contribute to an understanding of the opioid crisis in Canada

\begin{tabular}{c}
$\begin{array}{c}\text { Surveillance projects } \\
\text { by organization }\end{array}$ Description \\
\hline
\end{tabular}

Health Canada

General population surveys $^{12}$

Laboratory Information Management System (LIMS); Health Canada's Drug Analysis Service (DAS) ${ }^{13}$

New Psychoactive Substance (NPS) Online Survey

Canadian Post-Secondary Education and Drug Use Survey (CPADS)

Canada Compuscript (CS) - Quintiles IMS

Canadian Surveillance System for Poison Information (CSSPI) ${ }^{14}$
Canadian Tobacco, Alcohol and Drugs Survey (CTADS), a telephone-based biennial survey to capture data on tobacco, alcohol and drug use. In 2015, CTADS used a sampling frame for household surveys that included cell-phone-only households for the first time. Samples include Canadians 15 years and older. The CTADS replaced the Canadian Tobacco Use Monitoring Survey (CTUMS) and Canadian Alcohol and Drug Use Monitoring Survey (CADUMS). Health Canada also uses the Canadian Student Tobacco Alcohol and Drug Survey (CSTAD) to target student populations from Grade 7 to 12 across Canada.

The DAS is responsible for testing suspected controlled substances seized by law enforcement agencies that submit exhibits for testing. The test results are entered into LIMS, along with information on when the exhibit was submitted to the laboratory. This allows for regular surveillance of substances including NPS and pharmaceutical drugs.

The NPS Online Survey is currently being piloted. Canadians 15 years and older who report having used an NPS at least once in their life are recruited through a thread posted on Bluelight, websites on illicit drugs and an innovative online survey that uses a broad random intercept method. The questions are designed to provide insight on the new substances and on substance use patterns in Canada.

CPADS started as a pilot study in March 2018. CPADS will collect information via online surveys distributed to students aged 17-25 enrolled in participating postsecondary institutions. The surveys are distributed via schoolaffiliated emails.

The CS is obtained from purchased data from Quintiles IMS, in which dispensed prescriptions of pharmaceutical products in Canadian drug stores are available.

CSSPI consists of 5 poison control centres across Canada that medical professionals and the public can contact to seek treatment advice. Health Canada is currently collaborating with CSSPI to obtain standardized cases and uniform data elements across the poison control centres. Poison centres control calls will allow for an estimated baseline data on opioid use and harm across the country, which could be helpful in the establishing a toxicovigilance system. 
TABLE 1 (continued)

Overview of the existing and new federal surveillance initiatives that contribute to an understanding of the opioid crisis in Canada

Surveillance projects

by organization

Description

New

Health Canada (continued)

Internet monitoring of new synthetic opioids can help capture information on drugis categorized "not for human consumption." Systematic online searches can identify Canada-based websites that are selling NPS, in order to help

Internet monitoring Canada better meet international reporting obligations, inform risk assessment and scheduling of NPS, and inform potential health warnings and prevention initiatives. Law enforcement would also be kept apprised of new substances they can expect to find in clandestine laboratories. Currently, monitoring involves a manual search of Canadian websites.

Losses and thefts ${ }^{15}$

This database collects information on the reported number of controlled substances that are missing (because they were stolen or have been lost) from a pharmacy, hospital, health care practitioner or licensed/registered dealer. This database could be an indicator of diversion of substances.

Canada Vigilance Program The CVP collects information on adverse reactions from marketed products, including prescription opioids. Reports $(\mathrm{CVP})^{16}$ containing illicit drugs as suspect products are only captured in the database if a marketed health product is also mentioned as a co-suspect product.

Non-Insured Health Benefits (NIHB) Program ${ }^{17}$

NIHB is a national, medical health benefits program providing coverage for benefit claims for specified drugs and other medical items for eligible First Nations peoples.

Public Health Agency of Canada (PHAC)

Canadian Hospitals Injury Reporting and Prevention The CHIRRP collects information for harms associated with opioid use using data from emergency department visits in 11 sentinel pediatric and 6 general hospitals across the provinces and territories (excluding PEI, Saskatchewan, Northwest Territories and Yukon).

Program (CHIRRP) ${ }^{18}$

Track is an enhanced surveillance system that monitors the prevalence of HIV, HCV and other infections as well as

$\operatorname{Track}^{19}$ the associated risk behaviours (including opioid-related indicators) among injection drug users and men who have sex with men via venue-based sampling at a number of sites (most recently, 11) across Canada.

Opioid-related deaths and harms surveillance ${ }^{20}$

PHAC works closely with provinces and territories to collect data on apparent opioid-related deaths from their respective offices of chief coroners/medical examiners.

Canadian Institute for Health Information (CIHI)

National Prescription

Drug Utilization Information

System (NPDUIS) ${ }^{21}$

Hospital Morbidity

Database (HMDB) ${ }^{22}$

National Ambulatory Care

Reporting System (NACRS) ${ }^{23}$

Canadian Centre on Substance Use and Addiction (CCSA)

National Treatment

Indicators (NTI) ${ }^{24}$

The NTI project was established in 2009 to collect information on admissions to treatment centres, relying on a common set of treatment indicators on publicly funded programs across participating provinces and territories in Canada.

Abbreviation: NPS, new psychoactive substance.

changes, and identify patterns in risk factors that could be targeted by public health interventions.

\section{Surveillance: existing and new initiatives}

The Government of Canada recently established an Opioid Action Plan (OAP) ${ }^{9}$ for the implementation of immediate measures that will reduce largely preventable harms. As decisions and policies are to be based on sound, reliable information, there is an urgent need for robust, quality opioid-related data.

To address this gap, Health Canada recently implemented targeted surveillance initiatives that complement existing general population surveys. For instance, the New Psychoactive Substances (NPS) Online Survey is currently being piloted to determine if the presence, demand for and use of new psychoactive substance (NPS; including new synthetic opioids) could be accurately documented by reaching out to online communities of people interested in experimenting with psychoactive substances. The use of the term "new" does not necessarily indicate that these substances have been newly discovered or synthesized, but rather that they are newly available or have recently emerged as substances of misuse. During the pilot phase of the NPS Online Survey, Health Canada worked with administrators from a pre-existing online forum specifically 
designed for people with an interest in experimenting with psychoactive substances. This forum provides a platform where people who use drugs can exchange information and describe experiences, and discuss the psychoactive effects of substances they have tried. Forum participants are contacted and recruited through two methods: Canadians who report using an NPS at least once through a thread posted on Bluelight; and by casting an extremely broad, random intercept method using an innovative online survey.

The NPS Online Survey, which relies on voluntary participation to provide information on drug practices, is intended to contribute as an early-warning system about NPS in Canada-helping to document the presence, demand for and use of NPS in order to help meet international reporting requirements ${ }^{10}$ and to identify new substances of concern to inform control and regulatory processes.

In addition to novel surveillance approaches, the Opioid Data Working Group (ODWG) at Health Canada undertook a comprehensive review of existing opioid data holdings and surveillance activities in order to identify potential gaps and limitations. As the rise of harms associated with this public health crisis continues, the need to capture harmonized, national-level, in-depth data on risk factors will continue to grow. For example, in order to capture the current state of the opioid crisis in Canada, the Office of Drug Research and Surveillance (ODRS) is working with the Canadian Surveillance System for Poison Information (CSSPI) on harmonizing call data collected by poison control centres to capture details on drug-related harms, including due to opioid-related overdoses. The ODRS is also collaborating with its federal and provincial/territorial partners to determine how to best collate opioid- and other drug-related data from across Canada. Obtaining these data at a national level for Canada would be best achieved through the development and implementation of a Canadian Drugs Observatory, which is a best-practice model ${ }^{11}$ for ongoing nationallevel drug data collection, analysis and dissemination on drug use, harms and treatment. To this end, Health Canada is currently assessing the feasibility of implementing national drug observatory models.
Table 1 summarizes the existing and new federal initiatives that could efficiently provide useful information on behavioural and demographic risks, geographical patterns, harms and treatment.

\section{Conclusion}

Canadian public health stakeholders need better access to accurate and timely data to guide their response to the opioid crisis. A strong evidence base would enable public health actors to initiate an early warning system, identify trends, target interventions, monitor impacts and support decisions. While some measures of drug use, such as national-level surveys of the general population, are well-developed, other key data sources are not necessarily used in a way that makes a national rollup possible or to help inform and support the identification of risk factors for opioid misuse and associated harms. As such, novel approaches for collecting, analyzing and disseminating drug use data from surveillance activities will enhance the understanding of the availability, the associated patterns of use and the harms related to opioid use.

Finally, while several federal and/or provincial/territorial departments, along with non-governmental organizations, currently perform some of the functions typically associated with a drugs observatory, Canada lacks a dedicated national drugs observatory. Adopting this best-practice model for ongoing national-level drug data monitoring would help Canada respond efficiently to the opioid crisis and other emerging drug-related issues.

\section{Conflicts of interest}

All authors are employees of Health Canada. No author has any other conflict of interest to disclose.

\section{Authors' contributions and statement}

RA researched the information on federal programs. MJD conducted the IQVIA analysis. KA wrote this paper. SIP revised the paper. JL approved the final manuscript.

The content and views expressed in this article are those of the authors and do not necessarily reflect those of the Government of Canada.

\section{References}

1. Government of Canada. National report: apparent opioid-related deaths (2016) [Internet]. Ottawa (ON): Government of Canada; 2016 [cited 2018 Jan 16]. Available from: https://www.canada .ca/en/health-canada/services /substance-abuse/prescription -drug-abuse/opioids/national-report -apparent-opioid-related-deaths.html

2. Canadian Institute for Health Information. Opioid crisis having "significant” impact on Canada's health care system [Internet]. Ottawa (ON): CIHI; 2017 [cited 2018 Jan 16]. Available from: https://www.cihi.ca/en/opioid -crisis-having-significant-impact-on -canadas-health-care-system

3. Centers for Disease Control and Prevention. New research reveals the trends and risk factors behind America's growing heroin epidemic. [Internet]. CDC; 2015 July 7 [cited 2018 Jan 16]. Available from: https://www.cdc.gov /media/releases/2015/p0707-heroin -epidemic.html

4. International Narcotics Control Board. Narcotic drugs: estimated world requirements for 2013; statistics for 2011. New York: United Nations; 2012

5. Angus Reid Institute. Opioids in Canada: one-in-eight have family or close friends who faced addiction [Internet]. Vancouver (BC): Angus Reid Institute; 2018 Jan 11 [cited 2018 Jan 16]. Available from: http://angusreid .org/opioid-crisis/

6. IQVIA. Compuscript. 2017. Available from: http://www.imsbrogancapabilities .com/en/market-insights/compuscript .html

7. Canadian Institute for Health Information, Canadian Centre on Substance Abuse. Hospitalizations and emergency department visits due to opioid poisoning in Canada. Ottawa $(\mathrm{ON})$ : CIHI; 2016.

8. Bonevski B, Randell M, Paul C, et al.; Reaching the hard-to-reach: a systematic review of strategies for improving health and medical research with socially disadvantaged groups. BMC Med Res Methodol. 2014;14(1):42. doi: 10.1186/1471-2288-14-42. 
9. Canadian Centre on Substance Use and Addiction. Progress report on the joint statement of action to address the opioid crisis in Canada [Internet]. Ottawa (ON): CCSUA; 2017 [cited 2018 Jan 16]. Available from: http:// www.ccsa.ca/Resource \% 20Library /CCSA-Addressing-Opioid-Crisis-in -Canada-Summary-Report-2017-en.pdf

10. United Nations Office on Drugs and Crime. ARQ: Annual reports questionnaire. 2018 [cited 2018 Jan 16]. Available from: https://www.unodc.org/arq/

11. European Union. European Monitoring Centre for Drugs and Drug Addiction (EMCDDA). Building a national drugs observatory: a joint handbook. Luxembourg: EMCDDA; 2010.

12. Statistics Canada. Canadian Tobacco, Alcohol and Drugs Survey (CTADS). Detailed information for 2015 [Internet]. Ottawa (ON): Government of Canada; [modified 2016 Nov 11; cited 2018 Jan 16]. Available from: http://www 23 .statcan.gc.ca/imdb/p2SV

13. Health Canada. Drug analysis service [Internet]. Ottawa (ON): Government of Canada; [modified 2017 Nov 30; cited 2018 Jan 16]. Available from: https://www.canada.ca/en/health -canada/services/health-concerns /controlled-substances-precursor -chemicals/drug-analysis-service.html

14. Hosein S. The Canadian Surveillance System for Poison Information. CPHA; 2015 May 24 [cited 2018 Jan 16]. Available from: http://resources.cpha.ca /CPHA/Conf/Data/2015/A15-241e.pdf

15. Health Canada. Loss or theft report form [Internet]. Ottawa (ON): Government of Canada; 2014 [cited 2018 Jan 16]. Available from: https://www .canada.ca/en/health-canada/services /health-concerns/controlled-substances -precursor-chemicals/controlled -substances/compliance-monitoring /loss-theft-forgery/loss-theft-report -form.html

16. Health Canada. Canada vigilance program [Internet]. Ottawa (ON): Government of Canada; [modified 2016 Feb 19; cited 2018 Jan 16]. Available from: https://www.canada.ca/en/health -canada/services/drugs-health-products /medeffect-canada/canada-vigilance -program.html
17. Government of Canada. About the Non-Insured Health Benefits (NIHB) Program [Internet]. Ottawa (ON): Government of Canada; [modified 2016 Sep 1; cited 2018 Jan 16]. Available from: https://www.canada.ca/en /health-canada/services/non-insured -health-benefits-first-nations-inuit /administration/about-non-insured -health-benefits-nihb-program.html

18. Public Health Agency of Canada. Canadian Hospitals Injury Reporting and Prevention Program [Internet]. Ottawa (ON): Government of Canada; [modified 2016 Mar 9; cited 2018 Jan 16]. Available from: https://www .canada.ca/en/public-health/services /injury-prevention/canadian-hospitals -injury-reporting-prevention-program .html

19. Public Health Agency of Canada. I-Track overview and reports [Internet]. Ottawa (ON): Government of Canada; [modified 2014 Dec 10; cited 2018 Jan 16]. Available from: http://www.phac-aspc .gc.ca/aids-sida/about/itrack-eng.php

20. Apparent opioid-related deaths (March 2018) [Internet]. Ottawa (ON): Government of Canada; [cited 2018 May 25]. Available from: https://www.canada .ca/en/health-canada/services /substance-abuse/prescription -drug-abuse/opioids/apparent-opioid -related-deaths.html

21. Canadian Institute for Health Information. National Prescription Drug Utilization Information System metadata [Internet]. Ottawa (ON): CIHI; [cited 2018 Jan 16]. Available from: https://www.cihi.ca/en/national -prescription-drug-utilization-information -system-metadata

22. Canadian Institute for Health Information. Hospital morbidity database [Internet]. Ottawa (ON): CIHI; [cited 2018 Jan 16]. Available from: https:// www.cihi.ca/en/hospital-morbidity -database

23. Canadian Institute for Health Information. National Ambulatory Care Reporting System metadata (NACRS) [Internet]. Ottawa (ON): CIHI; [cited 2018 Jan 16]. Available from: https:// www.cihi.ca/en/national-ambulatory -care-reporting-system-metadata
24. Canadian Centre on Substance Use and Addictions. National treatment indicators [Internet]. Ottawa (ON): CCSUA; 2018 [cited 2018 Jan 16]. Available from: http://www.ccdus.ca /Eng/topics/Treatment-and-Supports /National-Treatment-Indicators/Pages /default.aspx 